INNOVACIÓN

\title{
Propuesta de sistematización de los elementos de la investigación jurídica. Resultados de una experiencia
}

\author{
Proposta de sistematização dos elementos da pesquisa jurídica. \\ Resultados de uma experiencia
}

Towards a systematization of the elements of legal research. Results of an experience

\author{
Fátima El Fakih Rodríguez iD \\ Universidad Católica de Temuco, Chile \\ Javier Enrique González Reinoza \\ Universidad Católica de Temuco, Chile
}

\begin{abstract}
RESUMEN En doctrina se acepta la afirmación de que el derecho se puede examinar desde tres categorías epistemológicas: la normativa, que lo reconoce en tanto regla de conducta; la factual, que da cuenta de la comprensión del derecho como un hecho social; y la valorativa, que entiende el derecho a modo de un sistema de valores según los cuales las normas fundamentan su esencia. Cada una de estas áreas carga la tinta sobre un modelo de investigación diferente, con elementos que varían según el área del saber jurídico en la cual se desarrolla. Como resultado de las disertaciones en una clase de investigación jurídica de un curso de primer año de la carrera de Derecho de la Universidad Católica de Temuco, surge una propuesta de sistematización teórica que intenta organizar la relación entre los elementos de la investigación jurídica y las áreas del conocimiento jurídico, logrando una herramienta didáctica para los estudiantes que comienzan a investigar, sobre la base de la importancia de la investigación como recurso epistemológico generador de conocimiento y facilitando el proceso de enseñanzaaprendizaje de los estudiantes. Esta investigación es de carácter conceptual, comparativa y sistemática.
\end{abstract}

PALABRAS CLAVE Investigación jurídica, enseñanza del derecho, elementos de la investigación jurídica, epistemología del derecho. 
RESUMO Na doutrina, a afirmação de que a lei pode ser examinada a partir de três categorias epistemológicas é aceita: os regulamentos que a reconhecem como regra de conduta; o factual, que explica a compreensão do Direito como um fato social e de valor, que entende o Direito como um sistema de valores segundo o qual as regras baseiam sua essência. Cada uma dessas áreas carrega a tinta em um modelo de pesquisa diferente, com elementos que variam de acordo com a área do conhecimento jurídico em que é desenvolvida. Como resultado das dissertações em uma aula de pesquisa jurídica, pertencente a um curso de primeiro ano da Faculdade de Direito da Universidade Católica de Temuco, surge uma proposta de sistematização teórica que tenta organizar a relação entre os elementos de pesquisa jurídica e as áreas do conhecimento jurídico, alcançando assim uma ferramenta didática para os alunos que começam a investigar, com base na importância da pesquisa como recurso epistemológico, gerando conhecimento e facilitando o processo de ensino-aprendizagem dos alunos. Esta pesquisa é conceitual, comparativa e sistemática.

PALAVRAS-CHAVE Pesquisa jurídica, educação jurídica, elementos de pesquisa jurídica, epistemologia jurídica.

\begin{abstract}
It is agreement in doctrine that the law can be examined from three epistemological categories: Normative, that recognizes it as a rule of conduct; the factual, it understands law as social fact and valorizes it, and another one that understands law as a system of values, according to which the norms fund its essence. Each of these areas emphasize a different model of investigation, with elements that vary according to the area of juridical knowledge in which it's developed. As a result of the dissertations in a class of the legal research branch, belonging to a first year course of the Law School of the Catholic University of Temuco, a systematization proposal arises that attempts to organize the relationship between the elements of legal research and areas of legal knowledge, thus achieving a didactic tool for students who begin to investigate, based on the importance of research as an e..pistemological resource generating knowledge and facilitating the teaching-learning process of students. This research is conceptual, comparative and systematic.
\end{abstract}

KEYWORDS Legal research, law education, elements of legal research, law epistemology.

\title{
Introducción
}

El proceso de investigación en el derecho es posiblemente más complejo que en otras disciplinas científicas. En las ciencias de la naturaleza como la física, la química o la biología, el investigador trabaja con premisas razonables, leyes o paradigmas a través de la formulación y ejecución de un procedimiento lógico que permite comprobar una hipótesis. En esos procedimientos la metodología es más o menos general y puede ser desarrollada según la naturaleza del problema investigado. Por otra parte, la 
discusión sobre la subjetividad de sus métodos o resultados no es relevante, sino que el investigador de estas áreas se siente seguro haciendo comprobaciones técnicas a sus procesos metodológicos para demostrar su cientificidad.

La mayoría de las ciencias sociales explicativas, en cambio, tienen un abanico de posibilidades metodológicas que proponen esquemas de percepción e interpretación de la realidad social que son acordados y pactados como correctos en el ámbito científico y que, eventualmente, pueden utilizarse en cualquier investigación según las necesidades de cada caso. En el derecho, el proceso investigativo también parte de un esquema de percepción de la realidad jurídica, acordada previamente en un marco que denominamos ordenamiento jurídico. Esto, si bien lo circunscribe a una realidad consensuada, no lo cierra ni aleja del contexto social en el que se da el derecho o en los elementos culturales en los cuales se inspira.

Los cambios generacionales en las aulas y en la academia en general en el proceso de enseñanza del derecho obligan a un replanteamiento. En un primer momento, se debe comprender que hay una diferenciación entre enseñar derecho y enseñar a investigar y hacer investigación jurídica. En segundo lugar, se debe comprender que la enseñanza de la investigación jurídica no puede hacerse sino sobre la base del mismo derecho, aunque este sea suficientemente amplio y complejo. En este caso el desafío es grande. Con las limitaciones cognitivas que supone el nivel básico del primer curso de carrera, aparece la necesidad de enseñar a investigar con el rigor científico oportuno ese derecho que el estudiante apenas comienza a conocer.

En este sentido, es de vital importancia reflexionar el papel que tiene sobre la enseñanza de las prácticas investigativas el conocimiento de los elementos del proceso científico, de manera que el estudiante no vea dicha tarea divorciada de las categorías ontológicas y cognitivas sobre las cuales ha venido estudiando y comprendiendo el derecho. Así, se propone tipificar la enseñanza de la investigación jurídica desde una perspectiva trialista y, a partir de ahí, plantear una sistematización teórica de los elementos del proceso de investigación jurídica, sobre la propuesta epistemológica del derecho que los alumnos de ese primer nivel conocen, desde el estudio de la teoría general del derecho o en la introducción al derecho.

Esta propuesta de esquematización teórica se concibe con la finalidad de facilitar el acercamiento de los alumnos del primer año de carrera al proceso de investigación científica del derecho. Busca contribuir a clasificar la investigación jurídica desde una concepción del derecho tridimensional. Es un artículo descriptivo y representativo, resultado de la experiencia en la programación y diseño del curso de investigación jurídica en los alumnos del segundo semestre del primer año de la carrera de Derecho en la Facultad de Ciencias Jurídicas, Económicas y Administrativas de la Universidad Católica de Temuco. 


\section{Las categorías epistemológicas o áreas del conocimiento jurídico}

En la jurisprudencia, entendida como la ciencia del derecho que se encarga del estudio de los fenómenos jurídicos, cada área que aborda tiene un acento o perfil metodológico distinto, en los que en la mayoría de los casos no es posible hacer pesquisas empíricas de los métodos utilizados para determinar definitivamente la cientificidad de los resultados, lo que podría ser considerado fundamento de crítica, subjetividad y relativismo. El nivel de complicación de la metodología jurídica se vería aumentado si no se comprendiera que no todas las ciencias del derecho deben ser consideradas metodológicamente iguales, ni que a todas se les puede aplicar de la misma forma iguales elementos en el proceso investigativo.

Cuando se enseña investigación jurídica a los estudiantes del primer año de la carrera que desean hacerse abogados, la gradación amplísima de elementos a tomar en cuenta y las grandes diferencias en su aplicación, dependiendo del área del derecho a aplicar, se nos presentan como un primer desafío.

En ese sentido, la enseñanza sistematizada de los elementos que componen la planificación de una investigación jurídica será fundamental para convertir la metodología del derecho en un instrumento aprovechable para los nuevos juristas y facilitar el uso de cada alternativa en el área o nivel de investigación que corresponda. De forma completa, esta sistematización pretende poner ante la vista del estudiante el conjunto de posibilidades de modo que puedan reconocer el área, los recursos y los diseños que proceden conforme al ámbito del saber del derecho en que se enmarcará cada problema a investigar.

Por otra parte, una de las mejores formas de presentar una información para ser comprendida es a través de un gráfico o tabla de doble entrada que permita la observación sencilla de la relación entre dos o más datos. Una de las dificultades que se puede presentar se encuentra en la necesidad de incluir información en un doble estándar, ya que un elemento de la investigación se puede repetir en algún área determinada, pero como se mostrará en las tablas finales, esos casos son excepcionales. La utilización de las tablas como herramienta estructural de ordenación de los elementos investigativos permite poner de manifiesto muchas regularidades entre el método y el área de la investigación, y que a su vez esta sea examinada y utilizada por los estudiantes en la tarea que comienzan a realizar.

Sosa Guerrero (1995: 25) explica cómo las categorías cognitivas conscientes e inconscientes son accesibles a la investigación científica y, en ese sentido, afirma que nacemos sabiendo el mundo en lo que de recurrente tiene. Así, los problemas de la metodología y la investigación jurídica consiguen ser resueltos por la inteligencia humana. Por demás, las acusaciones de subjetivismo, relativismo o sentimentalismo en la investigación del derecho estarían erradas porque no toman en cuenta que la metodología es una rama de la lógica que construye una especie de procedimiento 
operativo basado en el sentido común, entendido como una racionalidad mínima para formular, delimitar y resolver los problemas de la investigación jurídica. Este sentido común existe como piso para la investigación en el campo del derecho, indistintamente del área en que corresponda aplicarse. La selección de los elementos a investigar es un hecho objetivo que puede distinguirse desde un punto de vista racional y los elementos, según esto, pueden ser estandarizados. Por tanto, la tarea de sistematización es casi un deber.

Es un lugar común pensar que nuestro sistema perceptivo está condicionado por las convicciones, los sentimientos, la imaginación y los conocimientos previos entre otros aspectos. De todas formas, lo que percibimos lo acomoda la inteligencia de manera lógica creando posiciones conceptuales para entender la realidad. Lo podemos imaginar como una especie de casilleros lógicos en que la mente acomoda los objetos cognoscibles. Esos casilleros se encuentran vacíos y se pueden ocupar o no según la percepción de lo que hay en la realidad. Además, podrían ser los conceptos lógicos fundamentales que reflejen los vínculos y las conexiones entre el objeto y la inteligencia del sujeto. Estos casilleros son las llamadas categorías.

Aun cuando no hay acuerdo entre los filósofos del derecho sobre la naturaleza inherente de lo que se conoce como tal, síqueda claro que cualquier objeto de la realidad jurídica que se pretenda investigar puede ser considerado ontológicamente (en sí mismo) un valor, un hecho social (reflejado en la realidad social) o un fenómeno normativo (establecido en la norma que fija la pauta de conducta). Estas categorías epistemológicas, a partir de las cuales se puede conocer el derecho, son perfectamente ubicables bajo tres formas lógicas en tanto objeto cognoscente: derecho factum, derecho norma y derecho axioma-valor. Cada una de estas calidades del derecho constituye una organización del pensamiento particular, distinguible, según la cual se puede comprender cada fenómeno jurídico.

No hay que confundir las realidades en las que el derecho se presenta con las categorías epistemológicas a través de las cuales la mente jurídica clasifica y ordena el caos de las realidades fenomenológicas que se le muestran. La epistemología estudia dichas categorías que nacen como consecuencia de la determinación de esa realidad. Dada la realidad del derecho, la mente la conoce conforme a las categorías. Como expone Preciado Hernández (1981: 21-22), no podemos desconocer que el derecho tiene, en su entraña misma, una referencia a criterios racionales, de suerte que no se puede entender plenamente si no se le considera en relación con los valores jurídico-filosóficos que constituyen su causa final (Cano-Nava, 2011). El derecho es un producto del hombre y como consecuencia es inacabado, susceptible de ser creado y recreado en su esencia. Como la realidad del derecho es compleja, el número de categorías es múltiple, pero como ya se dijo toda realidad en la que se presenta el derecho es norma, hecho social o puramente un valor. 
En las investigaciones relativas al derecho se ha presentado la problemática de contar con teorías integrales que sean de aplicación práctica; requiere para su investigación de teorías complejas, que comprendan la totalidad de los elementos que la componen, por eso la teoría tridimensional del derecho del maestro Miguel Reale nos muestra la solución a ello (Cano-Nava, 2011).

El estudio del derecho y la investigación jurídica que sobre este se pretenda, por tanto, supone un análisis del derecho siempre dialéctico, una correlación permanente y progresiva a efecto de lograr la integración del hecho en valor dando origen a las normas. Es la forma en la que aprehende el objeto denominado derecho, considerándolo como un hecho o fenómeno que no existe sino en la sociedad y no puede ser concebido fuera de ella. Por tanto, el derecho tiene como cualidad inseparable el ser social (Cano-Nava, 2011).

El derecho es una ciencia que tiene por objeto el estudio de los fenómenos jurídicos que pueden presentarse en formas ilimitadamente diversas. Según Talamonti (2011, 153), así podría ser de interés para la jurisprudencia técnica el estudio de una ley, el comportamiento de los miembros de una sociedad en relación con dicha ley o el análisis de los valores que la misma regla encierra. Todos esos estudios son evidentemente de interés jurídico, pero leen el derecho de manera distinta, considerándolo desde el punto de vista normativo, sociológico o valorativo (Reale, 1997; García Amado, 1985). Para cada uno de estos casos hay tanto un diseño como un modelo de investigación diferente y, en general, una metodología que debe ser individualizada a cada área y, desde ahí, a cada problema a investigar. Esto es lo primero que deben tener claro los alumnos que comienzan a aprender a investigar el derecho.

No es pertinente generar pautas rígidas sobre los métodos, técnicas o patrones para la investigación del derecho. En cada caso específico el investigador debe atenuar, contextualizar y hacer dúctil la metodología, sus principios lógicos y modelos para diseñar una investigación particular, bajo la premisa de que a cada investigación le corresponde un proyecto de investigación, un modelo y un diseño de forma casi exclusiva. Esto también hay que aclararlo a los nuevos alumnos. De cualquier manera, cada área del derecho tiene sus propias especificaciones metodológicas que aquí se intentan clarificar y ordenar con la finalidad de escoger las herramientas para el diseño específico que debe realizarse en cada iniciativa investigativa.

Aunque sea subrepticiamente, casi cualquier reflexión sobre el derecho lo refiere como norma, vale decir, a un sistema de normas jurídicas con la propiedad de ser obligatorias para los destinatarios y exigibles por las autoridades, que son emanadas o reconocidas por un procedimiento formal. Las investigaciones en el campo normativo son de suma relevancia, ya que básicamente buscan determinar la vigencia de dicha norma, el contexto en el que se debe definir, interpretar, aplicar y la ubicación de ellas como ley positiva dentro de un sistema jurídico. Si un investigador, por ejemplo, se propone revisar el marco jurídico de protección de los pueblos indígenas en un 
país o en el derecho internacional, o pretende estudiar el procedimiento y las normas para constituir un contrato de arrendamiento por escritura pública en Chile, o examinar las pautas legales y jurisprudenciales del divorcio en Colombia, debe hacer una revisión de las normas sustantivas o adjetivas que involucran el aspecto a investigar y se encontraría viendo al derecho en su aspecto meramente normativo.

En los ejemplos anteriores el investigador indaga la realidad del derecho comprendiéndola como norma. Desde esta categoría epistemológica normativista, las investigaciones son técnicas y se encuentran en el área de la dogmática jurídica o ciencia del derecho en sentido estricto. Aquí los temas jurídicos se revisan desde las reglas: el sistema de relación entre ellas, su configuración como supuestos de hecho de una norma, sus formas de aplicación, la integración de los vacíos, las sanciones o consecuencias de su cumplimiento.

Los temas jurídicos que ameritan ser investigados desde el normativismo como categoría epistemológica pueden ser de una diversidad infinita, no son en número cerrado, ya que cada hecho de la realidad puede ser visto desde sus precedentes y consecuencias normativas. Incluso, muchos teóricos del derecho han afirmado que la ciencia del mismo debe reducirse a la indagación por lo normativo y que en última instancia solo debe estudiar los conceptos, categorías y figuras que ayuden a comprenderlo, excluyendo las fuentes valorativas y esencialistas del derecho y la realidad social a la que se aplica.

La corriente epistemológica que hace énfasis en las normas y sobre todo en las normas positivas como realidad del derecho es el iuspositivismo. Para la mayoría de los iuspositivistas, el derecho se reduce a los ordenamientos jurídicos de los Estados $\mathrm{y}$ arguyen que los derechos no se fundamentan en el derecho natural, sino en la autoridad de la que emanan conforme a un procedimiento formal que le dé validez. Para la corriente epistemológica del iuspositivismo, la validez del conocimiento jurídico nace de la adecuación del mismo a la norma y a la adecuación de esta al resto que conforma un sistema jurídico. Suponer bajo realismo ingenuo que el derecho es solo norma propone algunas limitantes, ya que una inmensa cantidad de los fenómenos no tienen una naturaleza normativa, por ejemplo, las instituciones que deben crear las normas, hacerlas cumplir, las relaciones sociales que intenta regular el derecho, el comportamiento de los funcionarios y las fuentes materiales, históricas, culturales y económicas que dan nacimiento a las reglas de derecho.

Si el investigador, por ejemplo, desea indagar sobre la eficacia de una norma, los grupos sociales y su influencia en la creación del derecho, la lucha de los grupos vulnerables o disminuidos por los poderosos para el reconocimiento de sus derechos, el impacto social de ordenes jurídicas, los grupos feministas, los delitos y las sanciones que deben aplicarse en un contexto social o las consecuencias de las migraciones para el derecho, la perspectiva normativista es insuficiente y debe enfocarse al derecho como una categoría sociológica. 
La categoría factual mira la realidad jurídica que se desarrolla en la sociedad, los procesos que generan derecho y cómo este último desencadena nuevos procesos sociales. La inmanencia social, histórica y política del derecho, los conflictos sociales y la división del trabajo como fundamento de las relaciones de producción, son algunos de los temas de las investigaciones factuales jurídicas. La corriente epistemológica más relevante que revisa el derecho desde esta categoría es el realismo jurídico, que se enfoca en los hechos y sobre todo en las actuaciones de los operadores jurídicos (Talamonti, 2011: 153). También los sociologistas, los institucionalistas y los relacionistas explican desde una perspectiva social al derecho. La disciplina jurídica fundamental que se encarga de estudiarlo como fenómeno social es, como se sabe, la sociología jurídica. La sociología del derecho indaga sobre las relaciones entre el derecho y la sociedad, sus relaciones recíprocas de transformación e incidencias de ambas disciplinas. El origen social del derecho, la esencialidad social de las normas y de las instituciones que la crean, interpretan, aplican y ejecutan.

Ahora bien, si la investigación se encamina a la revisión de la justiciabilidad de un hecho, conducta o fenómeno del derecho, su bondad social, la paz que el derecho debe significar para los miembros de una comunidad, la libertad que debe garantizar las reglas jurídicas y en general cómo esos valores de bondad, libertad y paz se deben recoger en las decisiones judiciales y en el ordenamiento jurídico, se investiga al derecho como categoría valorativa. En tanto que posiblemente la justicia es el fin primario del derecho, la categorización del mismo como valor estaría fundamentado principalmente en la justicia.

La corriente filosófica y epistemológica que estudia al derecho como valor es la teoría de la justicia o axiología jurídica, que tiene como objeto de estudio los valores en tanto se relacionan con el derecho. Las investigaciones jurídicas que se propongan en este campo, el ámbito axiológico, son eminentemente teóricas y se desarrollan en tres temas fundamentales: la existencia de los valores jurídicos, la posibilidad de conocerlos y la relaciones entre los valores, y el derecho como fundamento de los derechos fundamentales.

Como se explicó anteriormente, un problema a investigar puede tener distintas perspectivas de estudio, por ejemplo, el delito de hurto se puede estudiar como categoría normativa penal o como tipo penal para una investigación normativista, que intente explicar las consecuencias jurídicas y las penas de quien haya realizado la conducta prescrita como ese delito. Sin embargo, también se puede examinar como categoría factual examinando, por ejemplo, el número de hurtos que se suceden en un lugar determinado y también se pueden investigar los bienes jurídicos que se protegen al tipificar el hurto como delito. De cualquier forma, los problemas de investigación jurídica se pueden enmarcar en cualquiera de las categorías cognitivas del derecho: factual, axiológica o normativa. 
Tabla 1. Desde las categorías epistemológicas hacia las áreas de conocimiento del derecho.

\begin{tabular}{|ccccc|}
\hline $\begin{array}{c}\text { Categorías } \\
\text { cognitivas } \\
\text { universales }\end{array}$ & $\begin{array}{c}\text { Categorías } \\
\text { epistemológicas } \\
\text { del derecho }\end{array}$ & $\begin{array}{c}\text { Corrientes } \\
\text { cognoscitivas } \\
\text { del derecho }\end{array}$ & $\begin{array}{c}\text { Disciplina } \\
\text { jurídica } \\
\text { encargada }\end{array}$ & $\begin{array}{c}\text { Áreas } \\
\text { de conocimiento } \\
\text { del derecho }\end{array}$ \\
\hline Concepto & $\begin{array}{c}\text { El derecho como } \\
\text { «norma» }\end{array}$ & lus positivismo & Dogmática jurídica & Normativa \\
\hline Objeto & $\begin{array}{c}\text { El derecho como «hecho } \\
\text { social» }\end{array}$ & Realismo jurídico & Sociología jurídica & Factual \\
Sujeto & El derecho como «valor» & lus naturalismo & Filosofía jurídica & Valorativa \\
\hline
\end{tabular}

\section{Los elementos de una investigación}

Con lo explicado hasta ahora queda explícito que el objeto de la investigación jurídica está irremediablemente enmarcado en el estudio de alguno de estos tres elementos: norma, hecho social y valor. Así, con respecto a la norma o dogmática jurídica, se suele decir que bajo este prisma se investiga lo que los hombres dicen que hace el derecho. En cambio, del hecho o conducta social impetrada se dice que bajo esta óptica se investiga lo que los hombres hacen prácticamente con el derecho. Con respecto a la variable axiológica o valorativa, se suele afirmar que sobre la norma y el hecho social regulado hay valores o intereses protegidos que entrecruzan el discurso normativo y su vivencia sociohistórica (Witker, 2008: 953).

El interés por cuestiones didácticas y la propia variabilidad metodológica en la universidad, se han visto favorecidas por el cambio hacia un enfoque educativo centrado prioritariamente en el aprendizaje autónomo del alumnado, en el desarrollo de competencias y el aprendizaje a lo largo de su vida (Jiménez, González y Tornel, 2019), pero sin dejar de enriquecerse en la relación bidireccional entre docente y estudiante.

Así, si la enseñanza del derecho está interpelada a reformarse, esta necesidad imperiosa de renovación se refiere no solo a los contenidos que deben actualizarse de forma constante y periódica, sino también y principalmente a los modos de enseñar y a las formas de aprender las ciencias jurídicas (Cicero, 2018). Si los profesores no intentan conocer y llegar al nuevo tipo de alumno, si no hay una real comunicación entre ambos y tanto profesores como estudiantes discurren en distintas sintonías, se hará muy difícil sostener una relación pedagógica verdadera. La existencia de este vínculo es indispensable para sostener los aprendizajes y hacerlos profundos.

La metodología de la investigación jurídica consta de varios elementos fundamentales como la elección del tema, el proyecto de la investigación, el marco jurídico y conceptual, los métodos de búsqueda y análisis, y las fuentes de la información, conjuntamente de lo que en teoría se suponen suficientes argumentos de la verdad: deductivos que derivan conclusiones ciertas de premisas del sentido común (López, Ruiz, en Marín, 2014). Esto, que se traduce en un abanico de variables a ser maneja- 
dos por el nobel investigador al momento de ponerse en frente de una investigación jurídica, supone la necesidad de una visión interdisciplinaria a través de la cual logre una configuración de dicha complejidad de elementos, logrando una investigación más multifacética, de combinados compendios y mixtos factores como se verá en las siguientes líneas.

Si bien la aspiración final de esta investigación es lograr una sistematización de los elementos de la investigación jurídica en función de cada área de conocimiento del derecho, se han de resaltar previamente algunos caracteres definitorios de cada una de estas variables a tomar en cuenta en el momento en que el estudiante quiera emprender una investigación jurídica. La amplia bibliografía existente en relación con la metodología de la investigación jurídica pone especial atención en clasificar los elementos de la investigación en función de distintos parámetros y criterios.

Sin desechar la relevancia de todos esos criterios, se parte de una clasificación generalísima tomando en cuenta el carácter externo o interno al mismo proceso investigativo. Visto así, son considerados como elementos externos los que se han de determinar antes de alistarse propiamente en la investigación jurídica y que suponen la demarcación del tipo de investigación, el nivel, el enfoque y la función expectativa que se pretende cumplimentar con la investigación. Se llaman elementos internos los que pertenecen al desarrollo mismo de la investigación, que más allá del marco desde el cual se deslinda la investigación jurídica, incluyen los elementos intrínsecos al proceso investigativo, como son los pasos y herramientas determinados para ella.

\section{Los elementos externos de la investigación científica en el campo del derecho}

Los elementos externos de la investigación jurídica se entienden como las características que de forma exterior, es decir, desde afuera de la propia investigación, van a determinar el tipo, el enfoque, la función y el nivel de la investigación jurídica en virtud del área de conocimiento desde la cual se aborda y se plantea la pesquisa. Así, en un primer momento se ha de establecer si la investigación jurídica está orientada a resolver problemas de tipo teórico o práctico, encaminados a la refutación de hipótesis, a la producción, desarrollo, confirmación o confrontación de teorías.

\section{Según el tipo de investigación}

Cuando los autores hablan de investigación jurídica según el tipo, hacen referencia a la forma que puede adoptar dicha investigación de acuerdo con su objeto de estudio (Pérez, Pozo y Fernández, 2002). En la literatura sobre el tema se habla de tipos de investigación para identificar las diferentes formas que estas pueden adoptar en relación con aspectos diversos, cuestión que da lugar a una amplia y no unívoca taxono- 
mía (Villabella, 20012: 925). La perspectiva o categoría epistemológica desde la cual se haya planteado el abordaje de la investigación jurídica determinará su tipo entre teórica, empírica o mixta.

La investigación jurídica de tipo teórica se aplica sobre el estudio del derecho en abstracto, los objetos a investigar no se perciben sensorialmente, no son tangibles. Se entiende que es un prototipo de investigación especulativa, sobre ideas a defender, lo que se conoce como investigaciones de base cognitiva flexible (Guevara, 2013). La finalidad de este tipo de investigación jurídica, cognitiva, es la reconstrucción del núcleo teorético del derecho, es decir, la norma, entendida como forma lingüística elaborada por el hombre, un constructo racional y simbólico.

En ese sentido, se puede afirmar que adoptar un enfoque teorizado del derecho es casi una necesidad conceptual y práctica del razonamiento jurídico. En modo significativo los abogados usan una amplia red de significados, ideas, conceptos, narrativas o categorías incorporadas en teorías amplias, generales y complejas para poder practicar su profesión. Ahora, al mismo tiempo que resulta ciertamente un aprieto desatender el enfoque teorizado del derecho para poder practicar, ocurre que quien no asuma una perspectiva interna de la práctica operativa tendrá limitaciones insalvables para el ejercicio de teorización (Rojas, 2017).

La investigación jurídica de tipo empírica - o de campo- es la que aborda al derecho en tanto y en cuanto objeto. La fenomenología ocupa el desarrollo investigativo. Los procesos factuales del derecho, en tanto factor y producto social, se determinan a partir de rasgos, propiedades y/o manifestaciones concretas de hechos. Este tipo o modo de investigación jurídica busca la interacción directa del sujeto investigador con el objeto de estudio. Su aspiración última es establecer modelos de conducta, generalizaciones, dominando las cualidades intrínsecas del fenómeno jurídico de suerte que su comprensión sea el eje fundamental de dicho proceso. Ejemplo de este tipo de investigación jurídica, de estudio legal empírico, con un análisis descriptivo, sobre la base de datos legales empíricos inéditos que responda a la pregunta de investigación relativa al rol relevante, estratégico o meramente decorativo y que tiene referencias con el derecho comparado, se puede encontrar en la jurisprudencia del tribunal que desarrollan Alvés Marín y Vielma (2018).

Finalmente, se entiende como investigación jurídica de tipo mixta la que en un mismo proceso explora teoremas, conceptos, principios, fundamentos y/o leyes, y en ese mismo momento revisa empíricamente el objeto. Este tipo de investigaciones viene cundiendo cada vez más en los grupos de las escuelas de derecho, ya que es tendencia irreversible el hecho de que este último actúa holísticamente entre sus tres grandes campos cognitivos. Según el autor que se revise, el tipo de investigación jurídica puede estar determinado de una u otra forma de agrupación de los elementos. Por ejemplo, Ezequiel Ander Egg expone: 
Así, la tipología de la investigación guarda relación con variables como las siguientes: el formato de datos con el que se trabaje y los métodos que se requieren para interactuar con el objeto de las investigaciones (documental o de campo), el análisis de la información que realiza y el fin que se propone (cuantitativa y cualitativa), el nivel de profundidad a la que llega (exploratoria, descriptiva y explicativa), la dimensión de tiempo en la que se ubican (históricas, descriptivas-actuales, experimentales), el lapso de tiempo que abarcan (transversales u horizontales), el escenario en donde se desarrollen (de laboratorio, de campo o bibliográficas), la metodología que emplean (experimental, no experimental o cuasi-experimental), el propósito que tengan (pura y aplicada), etcétera (2003; 99).

\section{Según el enfoque de la investigación}

Lo que distinguirá a la investigación jurídica será la perspectiva que asuma el investigador frente al problema a investigar. Este elemento supone la delimitación de las propiedades y las variables del objeto que se estudie, en este caso, del derecho. Álvarez Undurraga se refiere en este sentido a la metodología y haciendo alusión a lo que aquí se llama enfoque, él expone que nos estaríamos centrando en el estudio de las diferentes corrientes del pensamiento jurídico, las que determinarán posiciones doctrinarias como el positivismo, el iusnaturalismo, el marxismo, etcétera (Álvarez, 2002: 30). El estudio de las diferentes corrientes del pensamiento jurídico determinará las posiciones doctrinarias, como las anteriormente mencionadas, que marcarán la orientación de la investigación. Este elemento de la investigación jurídica supone una determinada forma de análisis de los datos a investigar, una manera específica para tratar la información que se recaba, así como los métodos empleados y los razonamientos que se establecen a partir de ello. En este sentido, la investigación jurídica puede ser cualitativa, cuantitativa o multimodal.

La investigación jurídica de enfoque cualitativo suele identificarse con las preguntas ¿qué es? y ¿cómo es? Se tiende a precisar la cualidad del objeto a investigar, es decir, la manera de ser de ese objeto de derecho que se pretende estudiar, lo que lo distingue y lo caracteriza. Su propósito fundamental es la descripción del objeto del derecho que estudia, la interpretación y la comprensión del mismo. De ahí que se entienda que aun cuando su modelo de investigación se enmarque en los enfoques ius-naturalistas, humanistas, constructivistas y estructuralistas, es necesario hacer alusión al carácter fenomenológico desde el cual la investigación jurídica aborda problemáticas condicionadas, históricas y culturales en las que el hombre está insertado, y cuyo producto jurídico se forma.

La investigación cualitativa se relaciona con el paradigma interpretativo y tiene como objetivo tanto entender el objeto que estudia como evaluar las cualidades del mismo. Su propósito es destacar las relaciones y motivaciones subyacentes, por lo 
que utiliza un lenguaje fundamentalmente etnográfico, métodos que permiten la descripción y penetración de lo que estudia y muestras basadas en casos-tipo. Tiene la finalidad de interpretar, comprender y destacar las cualidades de lo que estudia (Villabella, 2012: 13).

La investigación jurídica de enfoque cuantitativo se relaciona con las preguntas ¿cuál?, ¿cómo?, ¿cuánto? y ¿dónde? Se corresponde con el paradigma positivista de la ciencia en general, que no se ha de confundir con la categoría epistemológica ius positivista del derecho. Este positivismo como enfoque supone que el conocimiento jurídico aquí desarrollado se basa en la experiencia de los sentidos y mira al derecho como un objeto cuantificable, observable, medible través de sus propiedades y manifestaciones. Con la finalidad de medir y verificar sus propuestas, se suele identificar con este enfoque a las investigaciones jurídicas que llevan hipótesis determinadas y sus resultados correlacionan así variables sobre las cuales explican nexos causales, de suerte que con ellas se logre establecer patrones de comportamiento nuevos, transformaciones de los existentes y nuevas generalizaciones (Villabella, 2012: 14).

La investigación cuantitativa está relacionada con el paradigma positivista de la ciencia y persigue como objetivo conocer el objeto de estudio a través de sus propiedades externas y manifestaciones observables, por lo cual se centra en recolectar datos del mismo, cuantificar magnitudes y hacer análisis porcentuales. Su propósito es la medición y demostración de las variables y relaciones que se han hipotetizado, para lo cual emplea un lenguaje esencialmente numérico, métodos que posibilitan la cuantificación y comprobación, y muestras representativas. Tiene la finalidad de establecer patrones de comportamiento, explicar, generalizar y predecir (Villabella, 2012: 14).

Se entiende que la investigación jurídica de enfoque multimodal conjuga los dos enfoques anteriores sobre un objeto en un mismo proceso investigativo. La postura epistemológica que sustenta este enfoque es el rebosado de la antítesis, entre los enfoques cualitativo (tesis) y cuantitativo (antítesis) (Hernández, Fernández, Baptista, 2003: 103; Machado Ramírez y De Oca, 2008). Cada vez es más común la aplicabilidad de este tipo de enfoque para todo tipo de investigación jurídica, ya que como se ha dicho, la tendencia es a visualizar el derecho como un sistema jurídico, un entramado de elementos (norma-hecho social y valores) y no parcelas aisladas actuando en separado. Esto representa la multilateralidad de los temas jurídicos que ocupan actualmente la investigación científica del derecho.

\section{Según el nivel de investigación}

Según el nivel de la investigación jurídica se puede distinguir una clasificación que algunos enmarcan en los mismos tipos de investigación. Muchos autores entienden que estos paralelismos entre niveles y tipología de investigación jurídica son posibles 
y plantean que puede ser: pura (descriptiva/explicativa), aplicada (descriptiva/explicativa/exploratoria) o mixta (descriptiva/explicativa/exploratoria).

El nivel de investigación jurídica pura se preocupa de elaborar teorías sin atender las posibles aplicaciones de estas. La investigación jurídica de nivel aplicada es la que se preocupa de utilizar las formulaciones teóricas elaboradas por la investigación pura para resolver problemas prácticos. $Y$ cuando se entiende que ambas se necesitan, en tanto están íntimamente conectadas, se habla de investigación jurídica mixta (Álvarez, 2002: 30).

Según estos niveles de investigación se puede incluir a su vez, en el mismo nivel de investigación jurídica pura, la investigación de nivel descriptivo y explicativo: una representación de lo que la norma en su texto quiere significar. En las investigaciones jurídicas de carácter aplicado, el nivel de la investigación además de descriptivo/explicativo puede ser exploratorio. Y las investigaciones jurídicas que se han clasificado como de nivel mixto ocuparán paralelismo en los caracteres doctrinal, descriptivo y explicativo.

En doctrina usualmente se entiende que los estudios exploratorios tienen como objetivo la formulación de un problema para posibilitar una investigación más precisa o el desarrollo de una hipótesis, pero además intentan aumentar la familiaridad del investigador con el fenómeno que se va a investigar y sondearlo. Los estudios descriptivos tienen como objetivo central exponer las características de los fenómenos, por lo tanto, deben medir una o más variables dependientes de una población definida o en una muestra de la población. Los estudios explicativos o causales están dirigidos a responder a las causas de los eventos físicos o sociales, luego su interés se centra en conocer por qué ocurre un fenómeno, en qué condiciones se da o por qué dos o más variables están relacionadas. Por lo tanto, son más estructurados y profundizados que los demás niveles de estudio (Álvarez, 2002: 32).

\section{Según la función de la investigación jurídica}

Al referirse a la investigación jurídica es necesario distinguir dos momentos previos en la actividad del jurista. Se trata de una primera etapa de investigación conceptual encaminada a la formulación de principios jurídicos, los que por su esencia misma rebasan los lindes del caso concreto, y de una segunda etapa que se desenvuelve en la aplicación de los principios utilizados en la solución de ellos. La primera es la esencia misma de la investigación jurídica y la segunda lo es del ejercicio profesional (Giraldo, 1985). A su vez, en ambos casos se puede distinguir que la función de la investigación jurídica, ateniéndose a su secuencia rigurosa, sin embargo, puede ocupar funciones distintas según sobre lo cual se ocupe. Puede ser sobre técnica jurídica o sobre fundamentos jurídicos. 
Entiéndase que la función determinada por la técnica jurídica subraya su atención en el conjunto de los medios jurídicos: tanto la formulación de la norma como la aplicación de ella por los prácticos. Esta permite la realización del derecho con una finalidad determinada. Su observancia permite un trabajo bien organizado y asegura resultados más fructíferos.

La función de la investigación jurídica demarcada sobre los fundamentos jurídicos atiende, en contrario, a los enunciados más generales, que estando o no integrados formalmente en los ordenamientos jurídicos particulares recogen de manera abstracta el contenido de un grupo de ellos y lo fundamentan. Se trata de una función tendiente a la investigación jurídica sobre conceptos y sobre las proposiciones de naturaleza axiológica.

Tabla 2. Elementos externos de la investigación jurídica

\begin{tabular}{|ccccc|}
\hline $\begin{array}{c}\text { Áreas } \\
\text { de conocimiento } \\
\text { del derecho }\end{array}$ & $\begin{array}{c}\text { Tipos } \\
\text { de investigación } \\
\text { jurídica }\end{array}$ & $\begin{array}{c}\text { Enfoques } \\
\text { de investigación } \\
\text { jurídica }\end{array}$ & $\begin{array}{c}\text { Niveles } \\
\text { de la investigación } \\
\text { jurídica }\end{array}$ & $\begin{array}{c}\text { Función } \\
\text { de la investigación } \\
\text { jurídica }\end{array}$ \\
\hline Normativa & Teórica & $\begin{array}{c}\text { Cualitativa/multimodal } \\
\text { Cuantitativa/ } \\
\text { multimodal }\end{array}$ & Pura & Sobre técnica jurídica \\
Factual & Empírica/Mixta & Cualitativa/multimodal & Pura & $\begin{array}{c}\text { Sobre técnica jurídica } \\
\text { Sobre los fundamentos } \\
\text { jurídicos }\end{array}$ \\
\hline
\end{tabular}

\section{Los elementos internos de la investigación científica en el derecho}

De las diferentes clasificaciones hechas por los autores acerca de los elementos de la investigación jurídica, se toman bajo la categorización de elementos internos todos los que sustentan hacia adentro el proceso investigativo. Visto así, se puede señalar que tanto los métodos como las técnicas de investigación dependerán del objeto de estudio que el investigador ha escogido y de la correspondencia dinámica, directa y dialéctica que debe existir entre la teoría y la práctica.

Esta categorización de los elementos que aquí se denominan hacia el interior de la investigación jurídica busca demarcar, a través de diferentes variables, el interior mismo del proceso investigativo. Se produce una diversidad de taxonomía en las distintas propuestas metodológicas de los autores, donde se encuentran categorizaciones de tipo documental o de campo; cuantitativa o cualitativa; exploratoria, descriptiva o explicativa; histórica, descriptiva-actual o experimental; transversal, longitudinal o transaccional; de laboratorio, de campo o bibliográfica; experimental, no experimental o cuasiexperimental; pura o aplicada, etcétera, que sin embargo, a los efectos de esta propuesta, se precisan en cinco grupos de elementos internos que serán abordados a continuación. 


\section{Según la forma de recoger la investigación}

En las investigaciones jurídicas, según sea el área de investigación escogida por el investigador, la forma del acopio investigativo variará considerablemente (Álvarez, 2002: 26). Distinguimos tres tipos: investigación documental, investigación de campo e investigación experimental.

La investigación documental fundamentalmente incluye la información recogida o consultada en documentos, así como en cualquier material impreso apto para ser analizado, procesado, analizado e interpretado.

En la investigación de campo el mismo objeto de estudio sirve de fuente de información para el investigador. Es la observación directa y vivaz de los fenómenos, de los comportamientos de personas y también de las circunstancias en las que ocurren determinados hechos importantes en la investigación jurídica. La naturaleza de esas fuentes va a determinar los modos de obtener los datos.

La investigación experimental, finalmente, es la descripción y análisis del objeto de estudio, lo que se espera que ocurra en condiciones esmeradamente controladas. El investigador maneja directamente las condiciones en las que se dan los fenómenos, poseyendo un control personal de la variable independiente, es decir, el papel protagónico del investigador determinará el curso de la investigación jurídica, en este caso, especialmente.

\section{Según los métodos utilizados en la investigación jurídica}

Suele entenderse al método como la vía para alcanzar una meta, es decir, una ruta a seguir de acuerdo con ciertos procedimientos que permiten investigar y obtener la comprensión de lo que se quiere investigar. Es la estrategia a través de la cual se investiga un problema científico-jurídico y se inquiere con ello en lo desconocido. Es un conjunto de instrumentos, técnicas y reglas mediante las cuales se produce el nuevo conocimiento. Su utilización «para el abordaje objetivo, crítico y causal de una contradicción científica y el hecho de que su empleo consciente y controlado posibilita que el conocimiento producido sea demostrable y comprobable, lo convierten en el eslabón fundamental de la ciencia» (Villabella, 2012). Técnicamente los autores lo identifican como un conjunto ordenado y sistematizado de instrucciones que facilitan la tarea de obtener nuevos conocimientos y de corregir los ya logrados.

En el ámbito de la investigación jurídica, el método debe tener similares características a diferencia de lo que puede llegar a ocurrir en la práctica profesional del abogado, donde la repetición de esquemas o del proceso de la búsqueda de soluciones a conflictos concretos, es decir, los planteados por el cliente, pueden llegar a convertir la labor en algo empírico y casi espontáneo, no tanto porque la tarea del litigio tenga esa naturaleza, sino porque la preparación profesional sigue creciendo en la actua- 
lidad de una adecuada vinculación con la práctica (Hernández, 1995: 18). En la literatura metodológic se distinguen tres métodos: exegético, sociológico y sistemático.

El primero de ellos, entendido como el método interpretativo por excelencia, ocupa el campo de la lógica formal, siendo una interpretación fundamentalmente gramatical, un método de interpretación deductivo del derecho que utiliza la investigación fundamental: técnicas, teóricas, dogmáticas, conceptuales, formales, etcétera. En segundo lugar, está la concepción de una investigación aplicada, que en el ámbito del derecho se refiere a la aplicación de técnicas de investigación llamadas humanísticas, sociológicas, realistas, que supone prácticas materiales del método sociológico (Durkheim,1985) sobre la base de la necesidad de registrar lo fenomenológico del hecho socio-jurídico en estudio. La investigación sociojurídica presenta el método sociológico como uno de los pilares fundamentales no solo de la investigación jurídica, sino también del quehacer profesional jurídico. Así, no se concibe el replanteamiento de casos prácticos en diversos escenarios de nuestra profesión que no se fundamenten en elementos empíricos indubitados, puestos en escena a través del método sociológico.

La enseñanza de este método y su aprendizaje constituyen tópicos priorizados de estos tiempos que cada vez ganan más espacios en los programas curriculares universitarios y tanto en eventos académicos como científicos de relevancia internacional (Aguilar, 2010). Finalmente, en el método sistemático se incluyen elementos que desarrollen el interés en la investigación de tipo valorativa a través de los postulados de la lógica informal que, según Atienza (1993), atiende a un proceso argumentativo sistemático, sustentado en la dialéctica y, más allá de la norma y de las instituciones que la aplican, se revisará el alcance de las mismas.

\section{Según la técnica utilizada en las investigaciones jurídicas}

La técnica de la investigación jurídica se entiende como el resultado del saber empírico-técnico que persigue la aprehensión de datos para el conocimiento sistemático, genético o filosófico del derecho, a cuyo efecto dota al sujeto cognoscente con todas las nociones y habilidades atinentes a la búsqueda, individualización y empleo de las fuentes de conocimiento jurídicas, de forma de obtener de ellas los datos concretos para su ulterior elaboración metodológica. Es un saber práctico al servicio de un saber científico. Las técnicas se refieren más a la operatoria de los medios auxiliares del método. Por otra parte, la técnica es un procedimiento particular al objeto de estudio y a la ciencia específica (Hernández, 1995: 18). En este sentido, se dividen en técnicas de investigación documental (interpretación/argumentación) y técnicas de investigación directa (observación, encuesta, muestreo y entrevista).

Las técnicas de la investigación jurídica se clasifican en dos grandes grupos: las técnicas de investigación documental o indirectas, que se dan en las investigaciones 
de tipo pura o básica y se caracterizan por el empleo predominante de todo tipo de documentos y técnicas de investigación de campo o directas, usadas en las investigaciones de tipo aplicada, en las que generalmente el investigador entra en contacto más estrecho con el objeto de indagar.

La técnica de investigación documental tiene por objeto capturar los conocimientos, experiencias y avances más significativos del fenómeno a investigar en el menor tiempo posible y con los resultados más satisfactorios. La adecuada instrumentación de las técnicas de investigación documental constituye el primer elemento indispensable para la formación del investigador. Las principales técnicas de investigación jurídica documental son la técnica de investigación bibliográfica, que implica complementariamente la habilidad en la compresión de la lectura en la mayor brevedad posible, y las de investigación hemerográfica, que se realiza en revistas especializadas y periódicos. Se hace la selección de los artículos vinculados con el objeto de la investigación y se registran en las fichas de contenidos directamente en el cuerpo de la investigación, destacándose las notas correspondientes.

En las técnicas de carácter meramente documental se incluyen las que siguen el método exegético, que se constriñen a la utilización de la lógica formal y las que siguen el método argumentativo como técnica basada en el proceso de lógica informal, que utiliza los razonamientos con el fin de conseguir sustentar una determinada tesis.

Las técnicas de investigación directa o de campo son complementarias de la técnica de investigación documental y también son llamadas técnicas de investigación social por sus aplicaciones en las ciencias sociales, algunos las han denominado técnicas empíricas. Mediante esta técnica es posible la captura de datos directamente de la fuente desde donde se generan, lo cual propicia una información directa con respecto al objeto de la investigación evitándose la triangulación que frecuentemente se da en los demás procedimientos. Las más conocidas y utilizadas son la observación científica (la más antigua y la más moderna de las técnicas para la investigación), y el testimonio oral y escrito conocido como la encuesta (recopilación de testimonios orales o escritos, provocados y dirigidos con el propósito de averiguar hechos, opiniones y actitudes, de la cual se entiende que debe incluir un trabajo documental previo siempre que exista la literatura disponible sobre el asunto. Los hechos, las opiniones y las actitudes son datos de gran valor para la investigación jurídica por sus evidentes implicaciones en las relaciones humanas).

Son otras técnicas de investigación jurídica de campo el muestreo, que se sustenta en la posibilidad de capturar información de aspecto parcial de un a totalidad o universo ante la imposibilidad de abarcar todo el universo; la entrevista, que se sustenta en la conversación entre entrevistador y entrevistado con condiciones previas como la programación, la previsión de las variables, su ejecución y consideración de los posibles entrevistados, así como de los instrumentos requeridos para la captura de 
datos; y la estadística, que tiene por objeto la evaluación de hechos, opiniones, experiencias, vivencias y de más fenómenos a partir de representaciones numéricas.

\section{Según los recursos a utilizar}

Para algunos autores, recursos e instrumentos son tomados como sinónimos, y frente a los métodos y las técnicas constituyen los elementos o categorías hacia dentro de la misma investigación jurídica. El instrumento de investigación es la herramienta que concreta el método, el medio a través del cual el investigador recolecta los datos y obtiene la información necesaria. Por ejemplo, la ficha de contenido que permite resumir la información existente en diferentes fuentes sobre un objeto al que se van a aplicar los métodos histórico-lógico y de análisis-síntesis, o la guía que estructura una entrevista (Villabella, 2012: 991). La investigación jurídica utiliza textos escritos y documentos que pueden ser leyes, jurisprudencia y doctrina, esta última cada vez más sistematizada de forma virtual en los distintos medios de publicación existentes. En las investigaciones jurídicas de carácter empírico, sobre los mismos recursos, el investigador amplía su gama a instrumentos de medición más específicos como las referidas en la categorización anterior y que son conocidas, por otros autores, como técnicas (encuestas, entrevistas, cuestionarios).

Tabla 3. Elementos internos de la investigación jurídica.

\begin{tabular}{|c|c|c|c|c|}
\hline $\begin{array}{l}\text { Áreas de } \\
\text { conocimiento } \\
\text { del derecho }\end{array}$ & $\begin{array}{l}\text { Según la forma } \\
\text { de recoger } \\
\text { la información en la } \\
\text { investigación jurídica }\end{array}$ & $\begin{array}{l}\text { Según el método } \\
\text { utilizado en la } \\
\text { investigación } \\
\text { jurídica }\end{array}$ & $\begin{array}{l}\text { Según la técnica } \\
\text { utilizada en la } \\
\text { investigación } \\
\text { juridica }\end{array}$ & $\begin{array}{l}\text { Según los recursos } \\
\text { utilizados en la } \\
\text { investigación jurídica }\end{array}$ \\
\hline Normativa & Documental & Exegético & $\begin{array}{l}\text { Documental: Interpre- } \\
\text { tación }\end{array}$ & Leyes, jurisprudencia y doctrina \\
\hline Factual & De campo/experimental & Sociológico & $\begin{array}{l}\text { Directa: Observación, } \\
\text { encuesta, muestreo y } \\
\text { entrevista }\end{array}$ & $\begin{array}{l}\text { Leyes, jurisprudencia y doctrina } \\
\text { + hechos observables }\end{array}$ \\
\hline Valorativa & Documental & Sistemático & $\begin{array}{l}\text { Documental: Argumen- } \\
\text { tación }\end{array}$ & Leyes, jurisprudencia y doctrina \\
\hline
\end{tabular}

Según las etapas de la investigación

No existe un consenso entre los diversos autores estudiados para señalar las etapas o los pasos a seguir en el proceso de la investigación jurídica, sin embargo, a los efectos de esta experiencia se tomó el texto del profesor Gabriel Álvarez como referencia y sobre la base de sus explicaciones se distinguieron dos (Álvarez, 2002: 25). 
Tabla 4. Sistematización de la investigación jurídica según los pasos de la investigación jurídica expuestos por Álvarez (2002)

\begin{tabular}{|ll|}
\hline $\begin{array}{l}\text { Áreas de conocimiento } \\
\text { del derecho }\end{array}$ & Según las etapas o pasos de la investigación jurídica \\
\hline Normativa & $\begin{array}{l}\text { 1. Delimitación del tema y planteamiento del problema } \\
\text { 2. Selección del diseño metodológico }\end{array}$ \\
3. Recolección de los datos obtenidos en fuentes documentales \\
4. Análisis e interpretación de los datos obtenidos en fuentes documentales \\
5. Exposición y presentación formal del trabajo de investigación
\end{tabular}

Etapas de la investigación jurídica de carácter documental

- Delimitación del tema y planteamiento del problema

- Selección del diseño metodológico

- Recolección de los datos obtenidos en fuentes documentales

- Análisis e interpretación de los datos obtenidos en fuentes documentales

- Exposición y presentación formal del trabajo de investigación

Etapas de la investigación jurídica empírica

- Delimitación del tema, planteamiento, formulación y sistematización del problema

- Objetivos generales y específicos, justificación y limitaciones

- Construcción del marco teórico y la hipótesis de investigación

- Selección del diseño metodológico de la investigación

- Recolección de los datos a través de las técnicas e instrumentos utilizados 
- Procesamiento, análisis e interpretación de los datos obtenidos

- Síntesis y presentación del trabajo de investigación, que tradicionalmente se da en un informe escrito y su exposición oral (Álvarez, 2002: 30)

Como se puede advertir, las etapas de la investigación jurídica de carácter dogmática corresponden a las de la investigación documental-bibliográfica, así como las etapas de la investigación empírica corresponden a las de la investigación de campo. El hecho de que exista esta distinción no impide que se combinen los tipos de investigación jurídica, permitiendo una mayor posibilidad de alcanzar aportes cualitativos y cuantitativos en el conocimiento del derecho (Bascuñan,1971; Fix-Zamudio, 1995; Álvarez, 2002: 3).

\section{Concluyendo una ordenación de los elementos de la investigación jurídica}

La clasificación de los elementos de la investigación jurídica planteada de acuerdo con las áreas del conocimiento del derecho supone una propuesta de estrategia integral y flexible, diseñada con la premisa de innovar y mejorar el aprendizaje de las diversas características y compendios del proceso de investigación científica para los estudiantes de derecho. En este sentido, surge como resultado de una aspiración de planificación didáctica en la que se conjugaron los conocimientos obtenidos previamente en los ramos de Teoría general del Derecho, Fuentes del Derecho e Interpretación y Argumentación Jurídica, con la expectativa de que esta adopción del esquema en cuestión se convierta en una útil herramienta para la enseñanza de la investigación jurídica.

La sistematización de los elementos de la investigación jurídica que aquí se presenta aparece como una suerte de saber pedagógico en la medida en que resulta de una construcción combinada entre docente y alumnos, en una práctica cotidiana, como efecto de un juego teórico-práctico. En ese sentido, como Maurice Ribeiro (2018) explica, la actividad docente no es solamente un objeto del saber independiente, sino que promueve una conjunción de diversos saberes que pedagógicamente se orquestan en doctrina, concepciones, reflexiones racionales y normativas que conducen a sistemas más o menos coherentes de representación y de orientación de la actividad educativa.

Finalmente, es importante referir que el modelo educativo de la Universidad Católica de Temuco se basa en competencias (Beca y otros, 2019). Estas pueden ser comprendidas y abordadas desde diversas perspectivas, siempre como el resultante de procesos complejos que los individuos ponen en acción-actuación-creación para resolver problemas y realizar actividades de la vida cotidiana y del contexto laboralprofesional. En ese sentido, fue precisamente a través de la puesta en práctica de estas competencias que se consiguió la automotivación, la iniciativa y el trabajo colabora- 
tivo de los alumnos, buscando el desarrollo (ser saber y saber hacer) de esta actividad de manera integrada, con la cual se pudo conseguir la categorización de los diversos elementos de la investigación jurídica y realizar la esquematización que acá se presenta desde una perspectiva trialista, como herramienta didáctica para la enseñanza de la investigación jurídica.

En este sentido, se presenta el siguiente cuadro sistemático como conclusión y en representación del objetivo final y didáctico de esta experiencia. Es importante resaltar que es resultado de la plenaria realizada en clases, de un trabajo previo de lectura y comprensión del material sobre los elementos de la investigación jurídica y de su desarrollo dentro de las aulas. Esta propuesta, por tanto, asume la tarea de cambio y planta cara al desafío actual de construir universidad, de la mano de una relación retroalimentativa signada por el desarrollo de actividades relacionales entre docente y estudiantes.

La presente sistematización opera como guía para abordar la extensa carta de elementos propuestos a seguir en la preparación de una investigación jurídica, de manera que a través del seguimiento de esta clasificación el estudiante maneje de forma más sencilla, didáctica y práctica a la vez, una serie de información que se encuentra dispersa en la bibliografía y constituya una ruta que le permita tanto planificar como llevar a cabo la construcción de proyectos de investigación.

Tabla 5. Modelo de sistematización de los elementos de la investigación jurídica

\begin{tabular}{|c|c|c|c|c|c|c|c|}
\hline 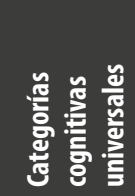 & 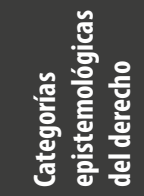 & 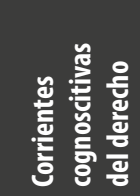 & 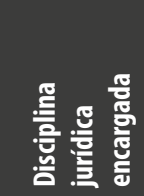 & 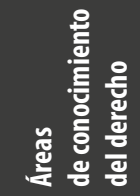 & 응 & 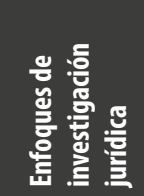 & 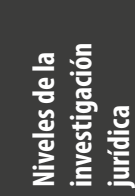 \\
\hline Concepto & $\begin{array}{l}\text { El derecho } \\
\text { como «nor- } \\
\text { ma» }\end{array}$ & $\begin{array}{l}\text { lus positi- } \\
\text { vismo }\end{array}$ & $\begin{array}{l}\text { Dogmática } \\
\text { jurídica }\end{array}$ & Normativa & Teórica & $\begin{array}{l}\text { Cualitativa/ } \\
\text { multimodal }\end{array}$ & Pura \\
\hline Objeto & $\begin{array}{l}\text { El derecho } \\
\text { como «hecho } \\
\text { social» }\end{array}$ & $\begin{array}{l}\text { Realismo } \\
\text { jurídico }\end{array}$ & $\begin{array}{l}\text { Sociología } \\
\text { jurídica }\end{array}$ & Factual & $\begin{array}{l}\text { Empírica/ } \\
\text { mixta }\end{array}$ & $\begin{array}{l}\text { Cuantitativa/ } \\
\text { multimodal }\end{array}$ & $\begin{array}{l}\text { Aplicada/ } \\
\text { mixta }\end{array}$ \\
\hline Sujeto & $\begin{array}{l}\text { El derecho } \\
\text { como «valor» }\end{array}$ & $\begin{array}{l}\text { lus natura- } \\
\text { lismo }\end{array}$ & $\begin{array}{l}\text { Filosofía } \\
\text { jurídica }\end{array}$ & Valorativa & Teórica & $\begin{array}{l}\text { Cualitativa/ } \\
\text { multimodal }\end{array}$ & Pura \\
\hline
\end{tabular}


Tabla 5. [Continuación]

\begin{tabular}{|c|c|c|c|c|c|}
\hline 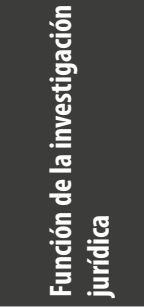 & 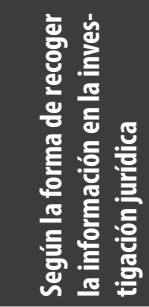 & 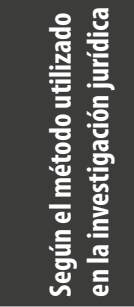 & 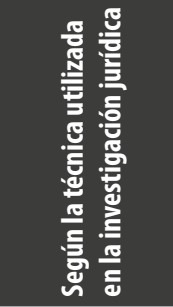 & 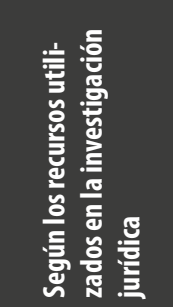 & 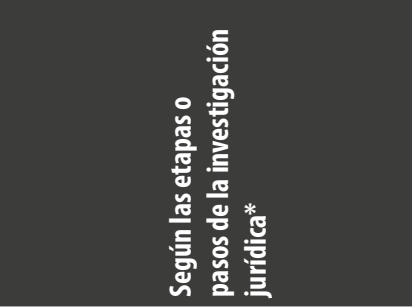 \\
\hline $\begin{array}{l}\text { Sobre técnica } \\
\text { jurídica }\end{array}$ & Documental & Exegético & $\begin{array}{l}\text { Documental: } \\
\text { Interpretación }\end{array}$ & $\begin{array}{l}\text { Leyes, jurispru- } \\
\text { dencia } \\
\text { y doctrina }\end{array}$ & $\begin{array}{l}\text { 1. Delimitación del tema y planteamiento } \\
\text { del problema } \\
\text { 2. Selección del diseño metodológico } \\
\text { 3. Recolección de los datos obtenidos en } \\
\text { fuentes documentales } \\
\text { 4. Análisis e interpretación de los datos } \\
\text { obtenidos en fuentes documentales } \\
\text { 5. Exposición y presentación formal del } \\
\text { trabajo de investigación }\end{array}$ \\
\hline $\begin{array}{l}\text { Sobre técnica } \\
\text { jurídica }\end{array}$ & $\begin{array}{l}\text { De campo/ } \\
\text { experimental }\end{array}$ & Sociológico & $\begin{array}{l}\text { Directa: } \\
\text { Observación, } \\
\text { encuesta, } \\
\text { muestreo y } \\
\text { entrevista }\end{array}$ & $\begin{array}{l}\text { Leyes, jurispru- } \\
\text { dencia y doctri- } \\
\text { na más hechos } \\
\text { observables }\end{array}$ & $\begin{array}{l}\text { 1. Delimitación del tema, planteamiento, } \\
\text { formulación y sistematización del pro- } \\
\text { blema. Objetivos generales y específicos, } \\
\text { justificación y limitaciones } \\
\text { 2. Construcción del marco teórico y la } \\
\text { hipótesis de investigación } \\
\text { 3. Selección del diseño metodológico de la } \\
\text { investigación } \\
\text { 4. Recolección de los datos a través de las } \\
\text { técnicas e instrumentos utilizados } \\
\text { 5. Procesamiento, análisis e interpretación } \\
\text { de los datos obtenidos } \\
\text { 6. Síntesis y presentación del trabajo de } \\
\text { investigación, que tradicionalmente se da } \\
\text { en un informe escrito y su exposición oral }\end{array}$ \\
\hline $\begin{array}{l}\text { Sobre los } \\
\text { fundamentos } \\
\text { jurídicos }\end{array}$ & Documental & Sistemático & $\begin{array}{l}\text { Documental: } \\
\text { Argumentación }\end{array}$ & $\begin{array}{l}\text { Leyes, } \\
\text { jurisprudencia y } \\
\text { doctrina }\end{array}$ & $\begin{array}{l}\text { 1. Delimitación del tema y planteamiento } \\
\text { del problema } \\
\text { 2. Selección del diseño metodológico } \\
\text { 3. Recolección de los datos obtenidos en } \\
\text { fuentes documentales } \\
\text { 4. Análisis e interpretación de los datos } \\
\text { obtenidos en fuentes documentales } \\
\text { 5. Exposición y presentación formal del } \\
\text { trabajo de investigación }\end{array}$ \\
\hline
\end{tabular}

* Las etapas o pasos de la investigación jurídica son tomadas de Álvarez (2002: 26-34). La sistematización de dichas etapas en función de las áreas de conocimiento del derecho es de elaboración propia. 


\section{Referencias}

Aguilar Avilés, Dager (2010). «Propuesta bibliográfica para el estudio de la metodología de la investigación socio jurídica». Contribuciones a las ciencias sociales. Disponible en https://bit.ly/2MaIEiR.

Álvarez Undurraga, Gabriel (2002). Metodología de la investigación jurídica: hacia una nueva perspectiva. Santiago: Universidad Central de Chile.

Álvez Marín, Amaya y Benjamín Vicente Vielma (2018). «Estudio legal empírico sobre el uso del derecho comparado por parte del Tribunal Constitucional de Chile». Revista de Derecho, 31 (2): 155-174. DOI: 10.4067/So718-09502018000200155.

Ander-EgG, Ezequiel (2003). Repensando la investigación-acción. Participativa. Buenos Aires: Lumen.

Atienza, Manuel (1993). Tras la justicia. Una introducción al derecho y al razonamiento jurídico. Barcelona: Editorial Ariel.

Bascuñán Valdés, Aníbal (1971). Manual de técnica de la investigación jurídica. $4 .^{\mathrm{a}}$ ed. Santiago: Jurídica de Chile.

BECA, Juan; Eduardo Castillo, Alejandra Cid, Eduardo Darritchon y Scarlette Lagos (2019). «Diseño de un examen de grado por competencias en la carrera de Derecho». Revista Pedagogía Universitaria y Didáctica del Derecho, 6 (1): 101-130. DOI: 10.5354/0719-5885.2019.53747.

Cano-Nava, Martha Olivia (2011). «Modelo epistemológico de la teoría tridimensional del derecho». Convergencia, 18 (57): 209-228. Disponible en https://bit. ly/2S64eIZ.

Cicero, Nidia (2018). «Innovar la enseñanza del derecho. ¿Solo se trata de tecnologías de la información y comunicación?». Revista Pedagogía Universitaria y Didáctica del Derecho, 5 (2): 91-109. DOI: 10.5354/0719-5885.2018.51976.

Durkheim, Emile (1995). Las reglas del método sociológico. Madrid: Akal.

Fix-Zamudio, Héctor (1988). Metodología, docencia e investigación jurídica. $4{ }^{\mathrm{a}}$ ed. Buenos Aires: Porrúa.

García Amado, Juan (1985). «Teorías del sistema jurídico y concepto de derecho». Anuario de filosofía del Derecho, 2: 297-316. Disponible en https://bit.ly/2PXWB4X.

Giraldo Ángel, Jaime (1999). Metodología y técnica de la investigación jurídica. $3{ }^{\mathrm{a}}$ ed. Bogotá: Librería del Profesional.

Guevara, Oscar (2013). Análisis del proceso de enseñanza aprendizaje de la Disciplina Proyecto Arquitectónico, en la carrera de Arquitectura, en el contexto del aula. Tesis doctoral de la Universidad Autónoma de Barcelona. Disponible en https:// bit.ly/2EsyaXQ.

Gutiérrez Pérez, José; Teresa Pozo y Antonio Fernández (2002). «Los estudios de caso en la lógica de la investigación interpretativa». Arbor, 171 (675): 533-557. Disponible en https://bit.ly/36PKKg1. 
Hernández, Sandra Luz y Daniel López (1995). Técnicas de la investigación jurídica. Ciudad de México: Harla.

Hernández Sampieri, Roberto, Carlos Fernández Collado y Pilar Baptista Lucio (2010). Metodología de la investigación. Ciudad de México: Mc. Graw Hill.

Jiménez Hernández, David, Juan González y María Tornel (2019). «Formación del profesorado universitario en metodologías y su incidencia en el aula». Estudios Pedagógicos, 44 (3): 157-172. DOI: 10.4067/So718-07052018000300157.

Machado Ramírez, Evelio y Nancy de Oca Recio, Nancy (2008). «Acerca de los llamados paradigmas de la investigación educativa: La posición teórico-metodológica positiva». Pedagogía Universitaria, 13 (1): 1-19. Disponible en https://bit. ly/38Tn7ow.

MARÍn Vichis, Rodrigo (2014). «Errores frecuentes en el uso de la metodología de la Investigación Jurídica». Revista Pedagogía Universitaria y Didáctica del Derecho, 4 (2). DOI: $10.5354 / 0719-5885.2017 .47972$.

Preciado Hernández, Rafael (1981). Lecciones de Filosofía del Derecho. Ciudad de México: Universidad Nacional Autónoma de México.

Reale, Miguel (1997). Teoría tridimensional del derecho: Una visión integral del derecho. Madrid: Tecnos.

Ribeiro, Adilson y Rita Buzzi Rausch. (2018). «Los saberes movilizados por el buen profesor en la visión de alumnos concluyentes del curso de derecho». Revista Pedagogía Universitaria y Didáctica del Derecho, 5 (1): 128-143. DOI: 10.5354/0719-5885.2018.50410.

Rojas MARroquín, Abdón (2017). «La fuerza ilocutiva y los efectos perlocutivos en el análisis estático de sentencias judiciales». Revista de Derecho, 30 (2): 9-30. Disponible en https://bit.ly/2sFlrOS.

Sosa Guerrero, Freddy (1995). Epistemología del arte. Ensayo de aproximación a una teoría del conocimiento estético. Mérida: Editorial Venezolana.

Talamonti, Paula (2011). «Sobre la posibilidad de generar conocimiento científico desde el campo del derecho». Revista Latinoamericana de Metodología de las Ciencias Sociales, 1 (2): 151-155. Disponible en https://bit.ly/2PBIzqB.

Villabella Armengol, Carlos Manuel (2012). «Los métodos en la investigación jurídica. Algunas precisiones». Disponible en biblioteca digital, Universidad Nacional Autónoma de México. Disponible en https://bit.ly/38PIKpO.

WITKER, Jorge (2008). «Hacia una investigación jurídica integrativa». Boletín mexicano de derecho comparado, 41 (122): 943-964. Disponible en https://bit.ly/2EydA8p.

\section{Sobre los autores}

FÁtima El Fakih Rodríguez es abogada, graduada por la Universidad de los Andes, Mérida, Venezuela. Además, es licenciada en Derecho y magíster scientiae en Socio- 
logía Jurídica por el Instituto Internacional de Sociología Jurídica (Oñati, España). Actualmente se encuentra en etapa de redacción y corrección de su tesis doctoral titulada «Límites del pluralismo jurídico. Una revisión al caso de Venezuela: ¿Fractura o aporía?» bajo la tutoría del doctor Marco Aparicio Wilhelmi en el programa de Doctorado en Derecho, Economía y Empresas de la Universitad de Girona, España. Es profesora emérita de la Escuela de Derecho de la Facultad de Ciencias Jurídicas y Políticas de la Universidad de Los Andes y se desempeña como profesora del Departamento de Ciencias Jurídicas en la Carrera de Derecho de la Facultad de Ciencias Jurídicas, Económicas y Administrativas de la Universidad Católica de Temuco. Su correo electrónico es felfakih@educa.uct.cl. (D) https://orcid.org/oooo-0002-3562-4417.

JaVier EnRiQue González Reinoza es abogado, graduado con honores Magna Cum Laude por la Universidad de los Andes, Mérida, Venezuela. Además, es magíster scientiae en Filosofía por la Facultad de Humanidades y Educación de la Universidad de Los Andes, Mérida, Venezuela. Actualmente se encuentra esperando fecha para su defensa de la tesis doctoral titulada: «La naturaleza, estructura y esencia de la norma jurídica. Una construcción de la teoría de la norma desde el derecho natural» bajo la dirección del doctor Jacinto Valdez Martínez en el programa de Doctorado en Derecho de la Universidad Panamericana de México. Actualmente se desempeña como profesor asociado de la Escuela de Derecho de la Facultad de Ciencias Jurídicas y Políticas de la Universidad de Los Andes, Mérida, Venezuela, y como profesor en la carrera de Contaduría de la Facultad de Ciencias Jurídicas, Económicas y Administrativas de la Universidad Católica de Temuco. Su correo electrónico es javierg@ula. ve. (D) https://orcid.org/0000-0001-6240-0171. 
La Revista Pedagogía Universitaria y Didáctica del Derecho (RPUDD) es una publicación científica semestral que contribuye a la reflexión multidisciplinaria sobre pedagogía universitaria y didáctica del derecho, para la formación y consolidación de esta área de investigación; así como a la difusión de prácticas innovadoras en la enseñanza-aprendizaje del derecho considerando el contexto nacional e internacional. Es una publicación electrónica internacional con una codirección entre Brasil y Chile.

\author{
DIRECTORA \\ María Francisca Elgueta Rosas \\ Universidad de Chile \\ DIRECTOR \\ Renato Duro Dias \\ Universidad Federal de Rio Grande, Brasil \\ SITIO WEB \\ pedagogiaderecho.uchile.cl \\ CORREO ELECTRÓNICO \\ rpedagogia@derecho.uchile.cl \\ LICENCIA DE ESTE ARTÍ́CULO
}

Creative Commons Atribución Compartir Igual 4.0 Internacional

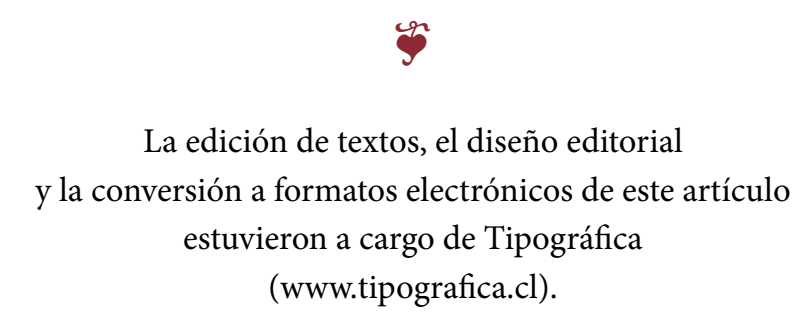

